\title{
Dietary management of heart failure: room for improvement?
}

\author{
Thomas Butler* \\ Department of Clinical Sciences and Nutrition, University of Chester, Chester CH1 4BJ, UK \\ (Submitted 4 August 2015 - Final revision received 18 December 2015 - Accepted 22 December 2015 - First published online 9 February 2016)
}

\section{Abstract}

There is growing awareness of the role of diet in both health and disease management. Much data are available on the cardioprotective diet in the primary and secondary prevention of CVD. However, there is limited information on the role of diet in the management of heart failure (HF). Animal models of HF have provided interesting insight and potential mechanisms by which dietary manipulation may improve cardiac performance and delay the progression of the disease, and small-scale human studies have highlighted beneficial diet patterns. The aim of this review is to summarise the current data available on the role of diet in the management of human HF and to demonstrate that dietary manipulation needs to progress further than the simple recommendation of salt and fluid restriction.

Key words: Heart failure: Diets: Remodelling: Cardiac function

Heart failure (HF) represents a clinically defined end point that can be the result of many different cardiac diseases that impair ventricular function. Impaired ventricular function results in clinical signs of disease such as dyspnoea, fatigue and oedema. HF can be classified based upon the time course of events, the side of the heart affected, whether systolic or diastolic function is impaired, ejection fraction (EF) and the severity of symptoms ${ }^{(1)}$. Mortality still remains high with $\mathrm{HF}$, although data from the UK National Heart Failure audit show that in-hospital mortality has fallen from $11 \cdot 1$ to $9.5 \%$ between $2011 / 2012$ and $2013 / 2014^{(2,3)}$. However, $6 \cdot 2 \%$ of patients who survive to discharge die in the $30 \mathrm{~d}$ following discharge, and overall 1-year mortality stands at $27 \%^{(3)}$.

In the UK, the most common New York Heart Association (NYHA) classification at the time of first hospital admission is class III or IV, representing a total of $80 \%$ of those diagnosed with $\mathrm{HF}^{(3)}$. Ischaemic heart disease (IHD) and hypertension (HTN) are observed in 46 and $54 \%$ of HF patients, respectively $^{(3)}$, suggesting that both conditions are important risk factors for the development of HF. Indeed, a medical history of IHD is more likely to result in the diagnosis of left ventricular (LV) systolic dysfunction and hence reduced EF, whereas HTN or valvular disease is associated with non-systolic HF with a preserved or normal $\mathrm{EF}(\mathrm{HFpEF})^{(3)}$. This latter form of $\mathrm{HF}$ is more frequently observed in obese women with pre-existing diabetes $^{(4)}$, whereas male sex, smoking and prior myocardial infarction (MI) are associated more strongly with HF with reduced $\mathrm{EF}(\mathrm{HFrEF})^{(5)}$. Recognised comorbidities present in the HF population include anaemia, cachexia, cancer, chronic obstructive pulmonary disease (COPD), depression, diabetes, gout, hyperlipidaemia, HTN, Fe-deficiency anaemia and renal dysfunction, all of which may require careful management in addition to the condition of $\mathrm{HF}^{(1)}$. Interestingly, those patients with HFpEF tend to have a higher non-cardiac comorbidity burden when compared with patients with $\mathrm{HFrEF}^{(6)}$, potentially identifying them as a unique patient group.

In addition to the known medical causes, HF has important socio-economical determinants. Individuals with HF living in the most deprived areas of the UK are more likely to present at a younger age when compared with those living in less deprived areas ${ }^{(3)}$, suggesting that additional factors - rather than just medical comorbidities - may influence prognosis. Such factors may include access to care, educational level but also lifestyle choices, including dietary habits.

The evolving knowledge of substrate usage in the failing heart has prompted several investigators to re-examine the importance of dietary modification in this patient group. This manipulation has extended further than preventing uncontrolled weight loss, itself shown to be linked with greater incidence of mortality ${ }^{(7)}$, to diet patterns linked with improvements in cardiac function and delayed mortality. It may be suggested that the window for nutritional intervention becomes narrower as HF progresses, with prevention of unintentional weight loss potentially more important in end-stage disease. Indeed, management of malnutrition and cachexia in HF patients is a key priority, and it has been reviewed extensively ${ }^{(8)}$.

There is a substantial gap in clinical guidance for the dietetic management of patients with $\mathrm{HF}$, despite widely recognised nutritional deficiencies ${ }^{(9)}$. Na restriction has been the significant

Abbreviations: DASH, Dietary Approaches to Stop Hypertension; EF, ejection fraction; FA, fatty acid; FAO, FA oxidation; HF, heart failure; HFpEF, HF with a preserved or normal EF; HFrEF, HF with reduced EF; HTN, hypertension; NYHA, New York Heart Association.

* Corresponding author: T. Butler, fax +441244 511310, email t.butler@chester.ac.uk 
nutritional recommendation by the American College of Cardiology Foundation/American Heart Association (ACCF/AHA) for the reduction of congestive symptoms ${ }^{(10)}$; however, this is not mirrored by European guidance ${ }^{(1)}$, itself providing limited advice other than of fluid restriction, maintenance of healthy weight and prevention of malnutrition. Irrespective of $\mathrm{Na}$, both guidelines provide little information into additional dietary changes that may be of benefit to the patient. The aim of this review is to present current developments in the understanding of nutrition in HF and to highlight the areas that need crucial development.

\section{Ventricular remodelling}

LV hypertrophy (LVH) is an important step in the development of HF. LVH may initially be beneficial in normalising wall stress and haemodynamic function ${ }^{(11)}$, and several animal models have suggested that inhibiting the initial hypertrophic process is detrimental $^{(12-14)}$. Pathological ventricular remodelling patterns have recently been shown to be associated with the incidence of $\mathrm{HF}$ and interestingly display differential risk for HF with HFpEF and $\operatorname{HFrEF}^{(13,14)}$. Specifically, individuals with eccentric remodelling have a greater than 2-fold risk of developing HFrEF, whereas those with concentric changes showed increased risk of HFpEF. These statistics are of significance given the high prevalence of HTN and IHD in HF patients ${ }^{(3)}$.

\section{Metabolic remodelling}

Ventricular remodelling processes also extend to metabolism and have been extensively reviewed ${ }^{(15-17)}$. Classically, the predominance of fatty acid (FA) oxidation (FAO) in the healthy heart is replaced by glycolytic substrate usage and reduced ability to utilise FA in the failing heart ${ }^{(17,18)}$, although this concept has been challenged ${ }^{(19)}$. Indeed, the conflicting changes observed in animal models may represent confounding factors such as the method used to induce HF, the strain of animal and duration of the intervention giving rise to different cardiac responses when challenged with varying diets ${ }^{(20)}$. Nonetheless, in patients with NYHA class IV HF, the mRNA and protein levels for key enzymes associated with FAO are reduced, supporting the metabolic change ${ }^{(21)}$. In addition to altered FAO, there is evidence that mitochondrial oxidation of glucose may be diminished in $\mathrm{HF}^{(18)}$, leading to a scenario in which the heart cannot process sufficient FA or glucose to maintain adequate energy supply. As such there is reduced ability to synthesise ATP leading to impaired contractile function. This concept of the failing heart being energy-starved is not new, and it is why the failing heart has been likened to 'an engine out of fuel' ${ }^{\text {,(22) }}$. Many groups have used this concept to suggest that manipulation of the diet to facilitate sufficient ATP production may be important in regulating function in the failing heart.

\section{The role of lipid in heart failure}

Much of the work on dietary manipulation has been performed in experimental models of $\mathrm{LVH}$ and/or $\mathrm{HF}$, and has been reviewed extensively ${ }^{(23,24)}$. A limitation of such models is that while providing useful mechanistic insight, they do little to represent benefits in quality-of-life and reduced rates of hospital admission. However, from these mechanistic studies, there is evidence to suggest that manipulation of nutrient intake predominantly carbohydrate and fat content - has an important role in regulating cardiac structure and function in $\mathrm{HF}^{(25)}$. The importance of fat is often overshadowed by its high energy content per gram; however, in HF patients, this same parameter may be beneficial in increasing an individual's energy intake and preventing unintentional weight loss and cachexia ${ }^{(8)}$. Several animal studies have also shown a potential beneficial role of dietary fat that extends beyond energy content, forcing us to question whether we should be encouraging a greater intake of this macronutrient in the HF population. For example, coronary artery ligation in Wistar rats has shown to reduce stroke volume and EF, although this finding can be partially attenuated by the provision of a diet containing $60 \%$ lipid ( $25 \%$ palmitic acid, 33\% stearic acid and 33\% oleic acid) ${ }^{(26)}$. This study also demonstrated that the high-fat diet had no impact upon cardiac performance in response to a dobutamine stress test, suggesting no additional impairment to contractile reserve. Equally, when failing hearts from rats fed a high-fat diet are perfused ex vivo, they demonstrate an improvement in cardiac FAO, which is similar to that of non-infarcted controls ${ }^{(27)}$. The authors of this study raise an important argument that following an MI, providing sufficient fuel for the non-infarcted myocardium is vitally important as the burden of function is often shifted to healthy tissue. This is further compounded by the observation that acutely limiting the availability of circulating FA in patients with cardiomyopathic HF depresses cardiac function, suggesting an important role of $\mathrm{FA}$ in $\mathrm{HF}^{(28)}$ (Table 1).

\section{Cardiac TAG and lipotoxicity}

The ability to store and utilise endogenous TAG has been shown to be important for cardiac function ${ }^{(29)}$, and the role of endogenous TAG is particularly important in the context of cardiac lipotoxicity. The traditional view of lipotoxicity relies upon the concept that a reduced capacity of the cardiomyocyte to oxidise FA coupled with normal or increased FA delivery leads to progressive lipid accumulation, the shuttling of FA species into the formation of biologically active intermediates such as diacylglycerol and ceramide, and ultimately cellular and organ dysfunction ${ }^{(30)}$. An excellent review on the role of FA and their derivatives as signalling molecules can be found in van Bilsen \& Planavila(31).

The traditional view of lipotoxicity being a pathology solely attributable to lipid accumulation is not completely accurate, and endogenous TAG accumulation may actually protect against biologically active intermediate formation with a specific role of various FA in this process. Indeed, previous research suggested that excessive supply of palmitate leads to increased apoptosis, and that provision of oleate in addition to palmitate can attenuate this by channelling palmitate into the formation of endogenous TAG and away from ceramide synthesis ${ }^{(32)}$. Although impressive, this study was performed in a cell culture 


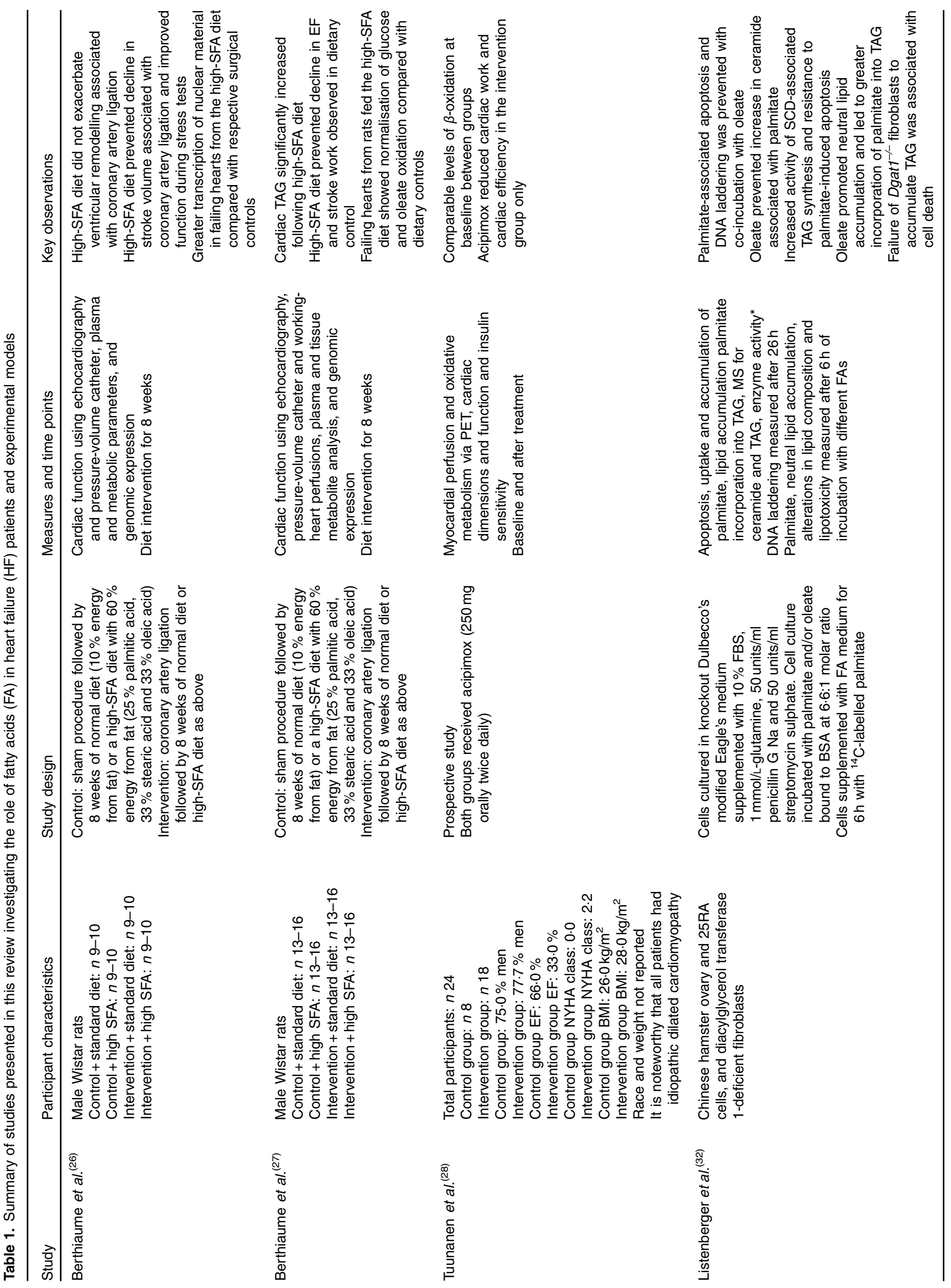


Diet and heart failure

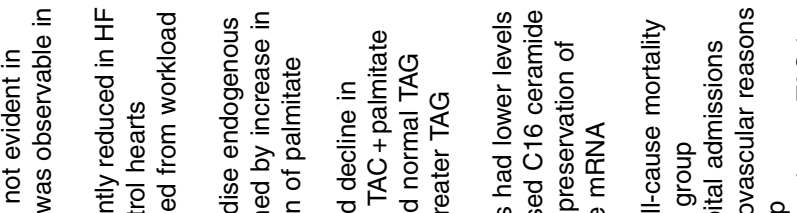

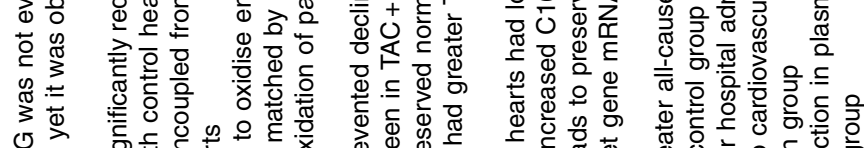

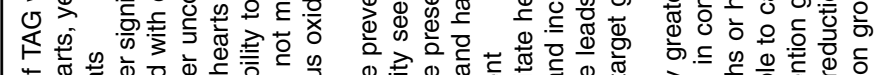

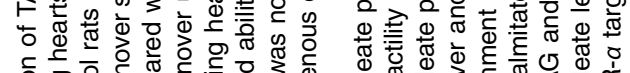

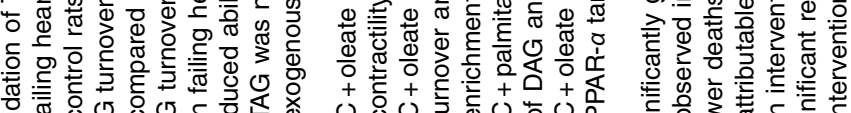

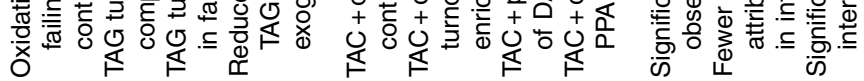

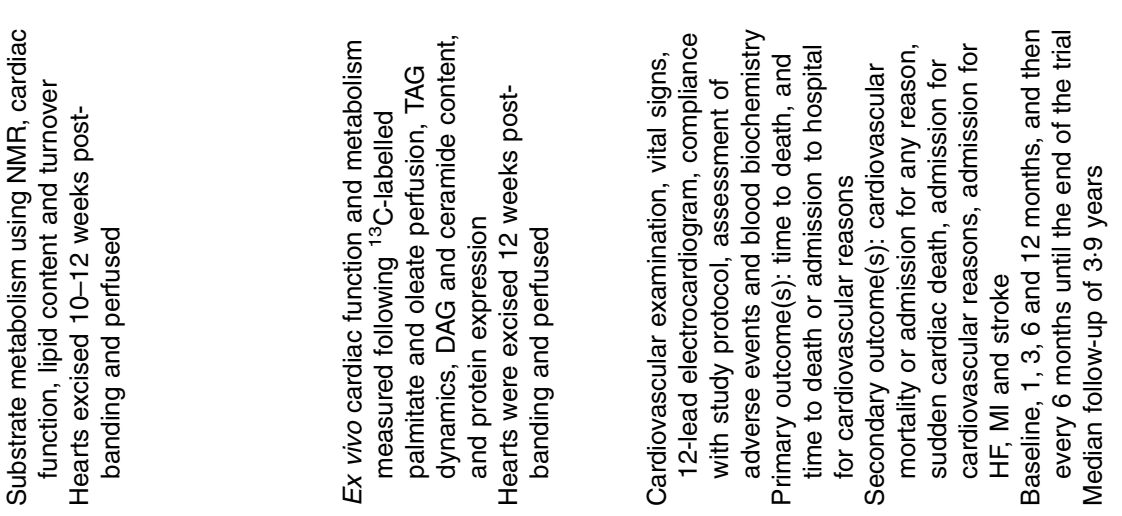

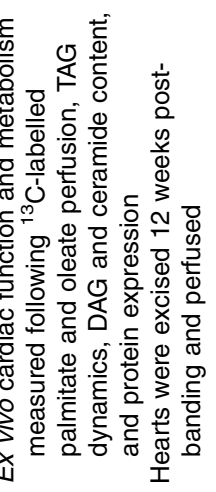

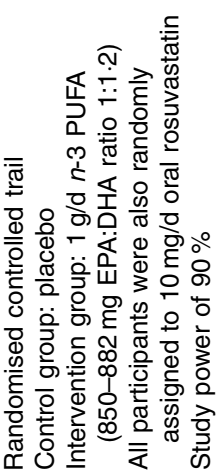

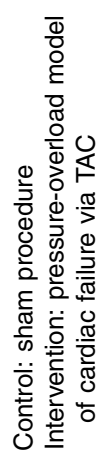

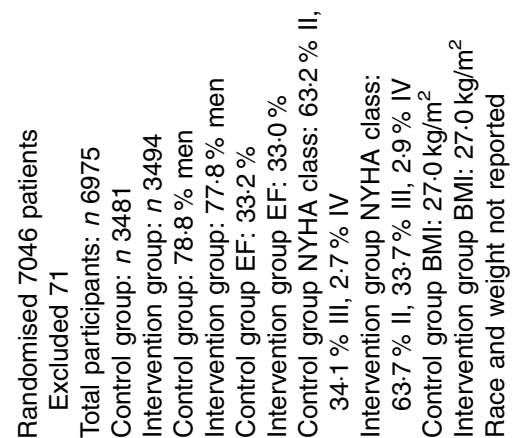

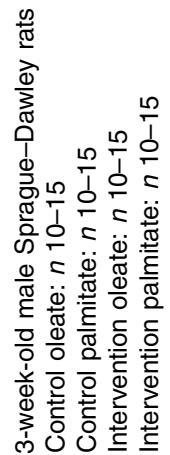

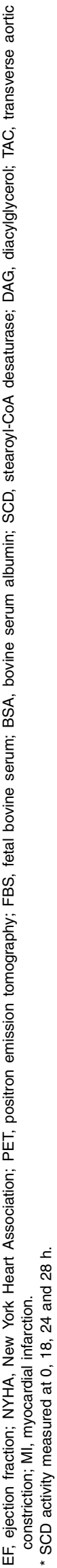


model, and it may not reflect the chronic nature of lipid accumulation in disease or the consequences of prolonged accumulation (Table 1). Nonetheless, it reflects the complexity of lipid dynamics ${ }^{(33)}$ and rasies questions over whether lipid accumulation per se is damaging, or wherther impairment to the dynamic nature of this energy store is more important.

In HF, endogenous TAG may be an important yet inaccessible source of substrate. The induction of HF in rats leads to a significant reduction in TAG turnover, suggesting impaired access to this energy store ${ }^{(34)}$. An inability to utilise stored TAG through decreased oxidation may lead to reduced energy provision in the setting of HF. Consequently, improving the heart's access to its own endogenous energy supply may have a significant impact upon cardiac function. In support of this theory, provision of oleate to failing hearts of Sprague-Dawley rats maintains the myocardial TAG pool and increases TAG turnover when compared with palmitate ${ }^{(35)}$. This finding was associated with improved cardiac contractility, augmentation of target genes associated with $\mathrm{FAO}$ and a reduction in the reactive intermediate $\mathrm{C} 16$ ceramide ${ }^{(35)}$. Although performed in rodents, the significance of this study is that by manipulating the exposure of the failing heart to different FA species mechanical performance can be improved (Table 1).

\section{$n-3$ Intake in heart failure}

n-3 Supplementation is currently listed as a class IIb recommendation and level B evidence in patients with systolic HF in European guidance $^{(1)}$, with similar recommendations present in ACCF/AHA guidance ${ }^{(10)}$.

The Gruppo Italiano per lo Studio della Sopravvivenza nell'Infarto Miocardico-Heart Failure study demonstrated the advantageous method of supplementing stage II-IV HF patients with $1 \mathrm{~g}$ daily of an EPA:DHA mix; however, it only produced a small yet significant reduction in hazard ratio for mortality compared with the placebo group ${ }^{(36)}$ (Table 2). A recent metaanalysis $^{(37)}$ has also confirmed the beneficial effect of $n-3$ on cardiac health and function in HF patients. In this study, pooled results of four studies totalling 350 participants showed fish oil supplementation to significantly reduce LV end-systolic volume compared with placebo. Similarly, analysis also suggested fish oil to be associated with improved $\operatorname{LVEF}^{(37)}$. Although this meta-analysis supports the notion that fish oil supplementation may have a beneficial effect in patients with HF, it remains to be determined whether similar effects can be observed by dietary sources alone.

Two systematic reviews and meta-analyses have been included to highlight the requirement of greater dietary research in $\mathrm{HF}$ patients. The first meta-analysis by Rizos et $a l^{(38)}$ considered randomised controlled trials whereby $n-3$ were administered to participants by supplementation or diet, with outcomes being all-cause mortality, cardiac death, sudden death, MI and stroke. The authors found no significant relationship between $n-3$ supplementation and measured outcomes, although a substantial limitation is evident when examining the dose of $n-3$ intake used in studies. Indeed, studies using a higher dose of $n-3$ supplement tended to show benefit, yet they themselves were limited by small sample size and therefore did not carry weight in the analysis. A more recent meta-analysis ${ }^{(39)}$ has also examined the relationship between $n-3$ and coronary risk as part of larger review of the relationship between all FA and coronary risk. The authors showed that $n-3$ supplementation was found not to be significantly associated with a reduced risk of coronary event in randomised controlled trials, whereas dietary $n-3$ intake was inversely associated with coronary outcomes in prospective studies. Indeed, this latter point is reinforced by the observation that a higher marine or dietary $n-3$ (EPA and DHA) intake is inversely associated with the development of $\mathrm{HF}^{(40)}$. It may be argued that if there is discrepancy in the dietary evidence base for the general population, is it safe and justifiable to offer the same advice to HF patients?

Considering all studies above regarding FAs, it is clear that the role of fat in HF is not as simple as once thought. Rather than focusing solely on the energy content of lipid, we should consider the biological and metabolic effects various FA may have, and use these to a potential therapeutic advantage. $n-3$ Supplementation may be of some benefit in HF patients, although it remains to be determined whether such benefits could be gained from increasing intake from dietary sources. At present, there are no recommendations for HF patients in terms of $n-9$ FAs, and thus it would be of use if appropriate studies were performed to examine the effects of increasing $n-9$ FA consumption in addition to $n-3$ in this patient group.

\section{Sodium and fluid restrictions in heart failure}

HF is characterised by altered renal perfusion, which itself leads to increased sympathetic activation and stimulation of the renin-angiotensin-aldosterone system (RAAS). Na and fluid are retained, leading to increased circulating volume in an attempt to preserve cardiac output. However, combined with fluid expansion, vasoconstriction caused by increased sympathetic activity raises blood pressure. Although initially beneficial, chronic activation of the RAAS and augmented $\mathrm{Na}$ and fluid retention increases both afterload and preload, contributing to oedema formation and congestive symptoms ${ }^{(41)}$. Reflecting the potential link between $\mathrm{Na}$ intake and fluid accumulation, the ACCF/AHA advise $\mathrm{Na}$ restriction in patients with symptomatic $\mathrm{HF}$, although this class of recommendation is IIa and carries a $\mathrm{C}$ level of evidence ${ }^{(10)}$. Fluid restriction to $1 \cdot 5-2 \cdot 5$ litres/d is also suggested by the ACCF/AHA in those patients with NYHA class $\mathrm{IV}^{(10)}$, in particular patients with hyponatraemia, with a similar recommendation by European guidance (although the latter carries no class or recommendation or level of evidence $)^{(1)}$. This is of concern given that $\mathrm{Na}$ and fluid restriction are viewed as a mainstay of dietary intervention in HF and is further complicated by the presence of 'salt-sensitive' phenotype, itself associated with increased mortality independent of blood pressure ${ }^{(42)}$.

Several studies have shown little clinical benefit in restricting $\mathrm{Na}$ and/or fluid, although these may be confounded by their acute setting $^{(43-45)}$ (Table 2). Compared with acute decompensated HF patients managed with a free-fluid regimen, acute 


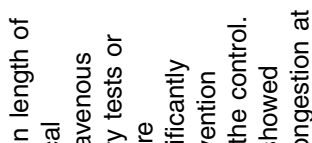

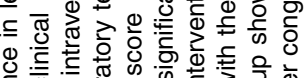

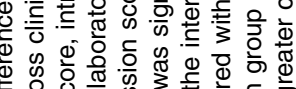

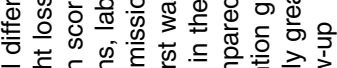

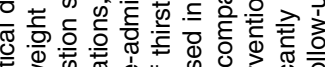

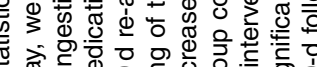

之

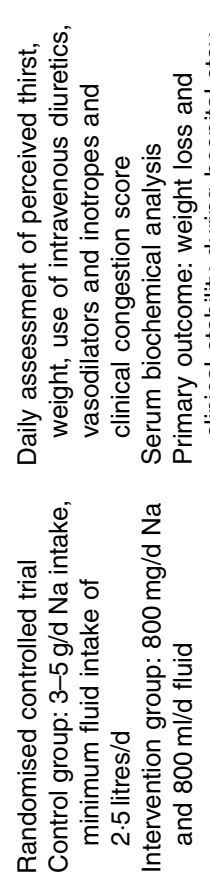

ส

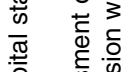

部

कृ

这

要

年

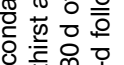

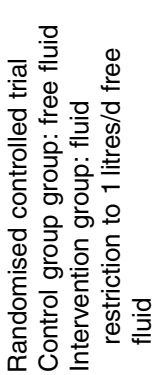

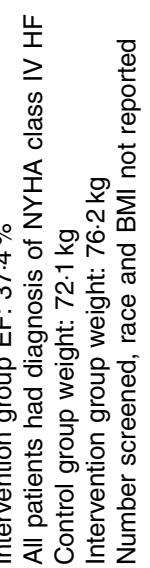

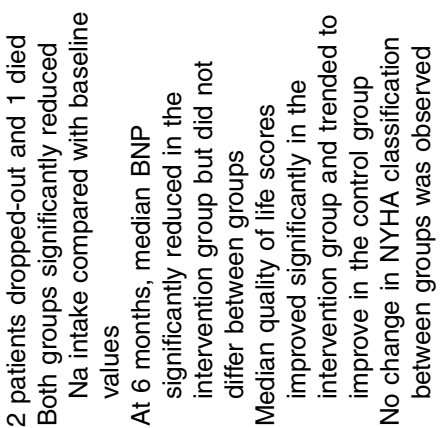

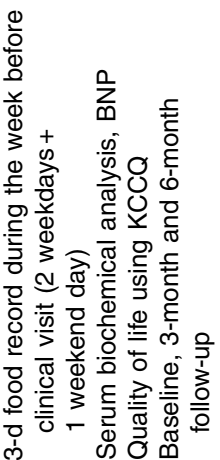

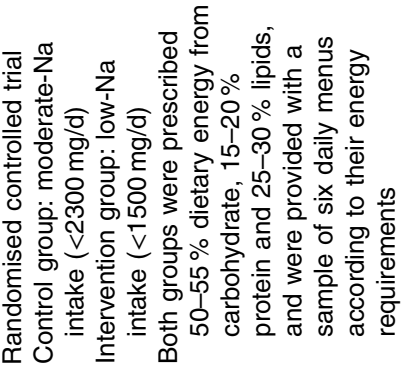




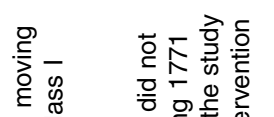

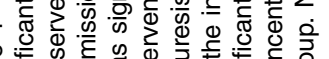
采

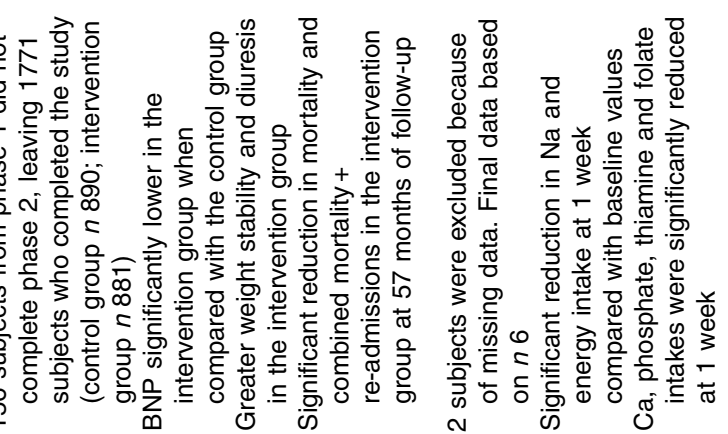

定

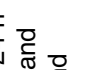

\section{(घ)}

$\varangle$

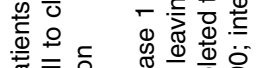

홍

등 응

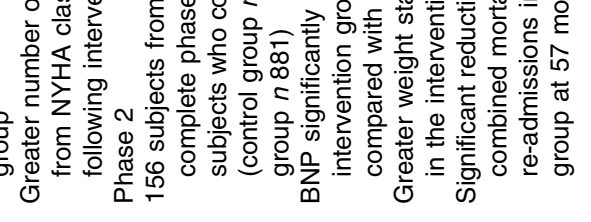

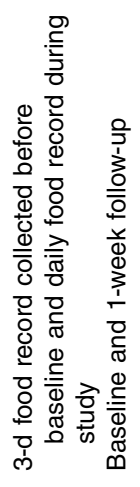

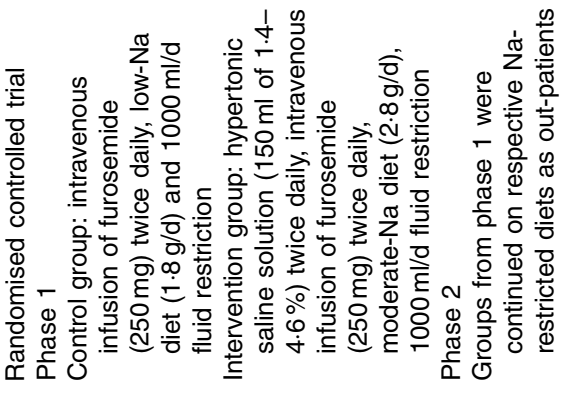

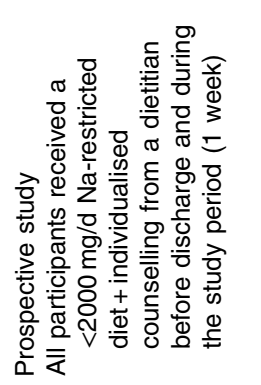

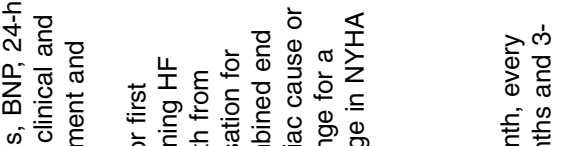

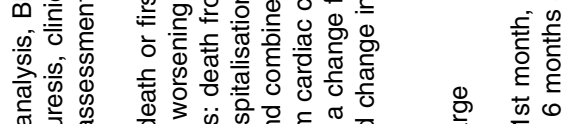

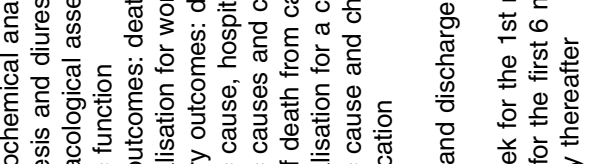

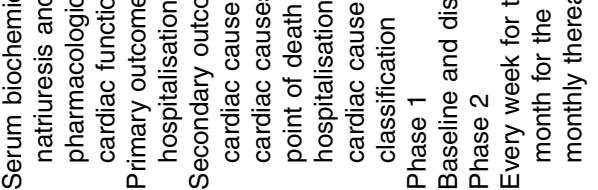

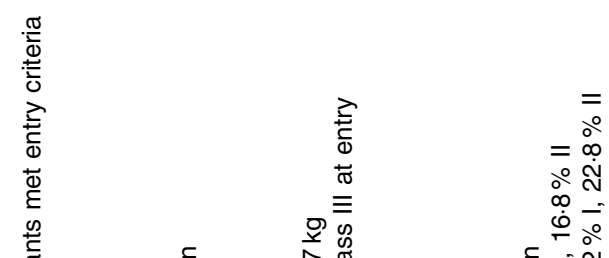

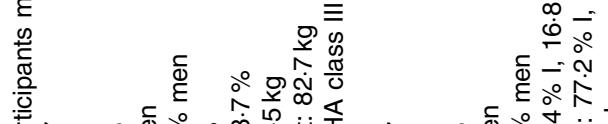

ฮั ฮิ

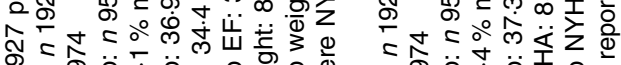

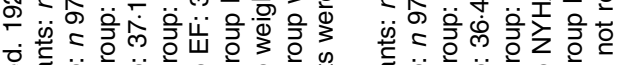

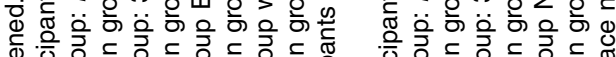

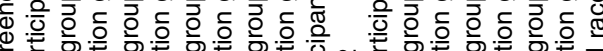


decompensated HF patients managed with fluid restriction showed no improvement in time to clinical stability or time spent receiving intravenous $\mathrm{HF}$ therapy ${ }^{(43)}$. An important limitation of this study is the difference in achieved fluid intake in both groups. In the free-fluid group, total daily fluid intake was $1466.6 \mathrm{ml}$ compared with $1074.3 \mathrm{ml}$ in the fluid-restricted group. Although it is statistically significant, clinically a greater restriction may have led to potential improvements; however, as the authors note, this may have increased thirst and reduced compliance. Similarly, a restriction of $\mathrm{Na}(800 \mathrm{mg} / \mathrm{d})$ and fluid intake $(800 \mathrm{ml} / \mathrm{d})$ in acute decompensated $\mathrm{HF}$ patients increased thirst and led to no improvement in 30-d hospital re-admission rates when compared with a control group receiving no such restriction ${ }^{(44)}$. Furthermore, levels of braintype natriuretic peptide (BNP) were significantly higher in the restricted group at the end of the study. A very real confounding factor in these trials examining $\mathrm{Na}$ restriction is their acute setting. Indeed, a low-Na $(1500 \mathrm{mg} / \mathrm{d})$ diet proved to be more effective at reducing BNP in ambulatory HF patients with NYHA II/III when compared with a moderate-Na $(2300 \mathrm{mg} / \mathrm{d}) \operatorname{diet}^{(45)}$. An important aspect of this study is the use of ambulatory HF patients as opposed to acute decompensated patients $\left({ }^{40,41)}\right.$.

To further complicate the issue of $\mathrm{Na}$ restriction in $\mathrm{HF}$ patients, a moderate in-hospital $\mathrm{Na}$ restriction $(2800 \mathrm{mg} / \mathrm{d})$ combined with hypertonic saline solution, $250 \mathrm{mg}$ twice daily intravenous furosemide and $1000 \mathrm{ml}$ fluid restriction in patients with HFrEF produced a greater improvement in diuresis and natriuresis when compared with a group of $\mathrm{HF}$ patients receiving a greater $\mathrm{Na}$ restriction $(1800 \mathrm{mg} / \mathrm{d})$ and no hypertonic saline solution. These patients were discharged on their in-hospital $\mathrm{Na}$ and fluid restrictions in addition to $50-125 \mathrm{mg}$ twice daily furosemide. Those who maintained the moderate$\mathrm{Na}$ intake showed reduction in the occurrence of the combined end point of mortality and hospital re-admission in comparison with the restricted group ${ }^{(46)}$. The authors of this study speculate that the greater $\mathrm{Na}$ intake during the hospital admission and discharge may improve serum Na levels, chronically reduce neuro-hormonal activation and improve delivery of diuretics to the loop of Henle, thus increasing their action of diuresis (Table 2)

It is also relevant to consider in the context of $\mathrm{Na}$ restriction that salt taste diminishes with age ${ }^{(47)}$, and that restricting $\mathrm{Na}$ in hospitalised HF patients may lead to an increased desire to satisfy the salt taste on discharge, further compounding difficulties of adhering to a low-Na diet. This concept would support the observations of Aliti et al. ${ }^{(44)}$. As such, consideration needs to be given to the different HF populations (ambulatory or hospitalised) in addition to the support required for patients to adhere to such a diet upon discharge. Without support, we are expecting a great deal from the elderly HF population, which may be an additional reason why low-Na diets are so difficult to follow. It would also be prudent to note that restricting $\mathrm{Na}$ intake in $\mathrm{HF}$ patients has been shown to be associated with reduced intake of other important nutrients such as $\mathrm{Ca}$, phosphate, thiamine and folate ${ }^{(48)}$, and therefore it would be advisable that patients discharged from hospital with low-Na advice receive regular follow-up to ensure compliance and also so that dietary adequacy can be reviewed (Table 2).
A recent Cochrane meta-analysis ${ }^{(49)}$ has suggested that $\mathrm{Na}$ restriction leads to increased plasma renin, aldosterone, adrenaline and noradrenaline, irrespective of whether the individual is hypertensive or not, and as such may aggravate features of decompensated HF and explain the outcomes in previously mentioned studies. Furthermore, elevated levels of plasma renin activity have been linked with increased mortality in patients with stable symptomatic HF NYHA class III-IV, irrespective of pharmacotherapy ${ }^{(50)}$. In the analysis by Graudal et al. ${ }^{(49)}$, the authors report that restriction of $\mathrm{Na}$ to a sub-normal level resulted in a 1 and $3.5 \%$ decrease in systolic blood pressure (SBP) in normotensive and hypertensive individuals, respectively. They also suggested that in normotensives a greater duration of $\mathrm{Na}$ restriction produced a larger reduction in SBP (estimated mean difference of $0.4 \mathrm{mmHg}$ ); however, the reduction in SBP following Na restriction in hypertensive individuals did not appear to be time-dependent. It may be inferred from these observations that $\mathrm{Na}$ restriction may have a greater impact upon afterload in those HF patients with co-existing HTN who are salt-sensitive. Although HTN is more common in those individuals with $\mathrm{HFpEF}$, it is not exclusive to this group, and therefore examining the specific benefits of low-Na diets in both hypertensive and non-hypertensive HFrEF and HFpEF populations would be of use.

Considering different responses to $\mathrm{Na}$ restriction between acute decompensated and compensated HF patients, in addition to those who may be more salt-sensitive, a welldesigned clinical trial comparing short and long-term effects of $\mathrm{Na}$ restriction is required not solely on the outcome of mortality but on additional clinically relevant factors such as quality of life and hospital re-admission. A key recommendation should be that any $\mathrm{Na}$ and fluid $\mathrm{Na}$ restrictions need be individualised based on the severity of $\mathrm{HF}$, dose of diuretic, degree of fluid accumulation and the clinical setting.

\section{Dietary patterns and disease progression in heart failure}

Discussion of the dietary management of each individual comorbidity experienced by HF patients is beyond the scope of this review. However, is the author's opinion that through appropriate nutritional education there is no reason why dietary patterns such as the Mediterranean or Dietary Approaches to Stop Hypertension (DASH) diet cannot be modified to account for comorbidities such as diabetes, COPD or gout, and act as an adjunct to traditional pharmacotherapy for these conditions in HF patients.

\section{Dietary Approaches to Stop Hypertension and Mediterranean diet}

Cohort studies have identified several dietary patterns as being cardioprotective. Famous examples include the Mediterranean and DASH diets ${ }^{(51)}$. A dietary pattern approach is important, as it acknowledges the synergistic effects of different foods, rather than focusing on a single nutrient, and recently studies have examined diet patterns in relation to specific outcomes in $\mathrm{HF}^{(52)}$. Higher intakes of salty foods are associated with a shortened 
time to transplantation in patients with advanced HF, and increasing the intake of foods rich in MUFA and PUFA from 'occasionally' to 'several times a week' was associated with approximately $50 \%$ reduction in risk of death/deterioration ${ }^{(52)}$. Other interesting results from this study include the association between different food groups. SFA was significantly associated with increased consumption of salty food, and inversely associated with MUFA and PUFA. Similarly, both MUFA and PUFA also positively correlated with fruits/vegetables/legume intake, thus suggesting that the consumption of one nutrient may predict other dietary components. This observation may be important for the clinician or dietitian when taking a diet history, and it may allow a more rapid determination of diet quality. However, although interesting, this study is limited by the use of the food FFQ and does not provide information on the amount of such nutrients consumed by the participants.

The DASH diet has a recognised beneficial effect in delaying the incidence of $\mathrm{HF}^{(53)}$, and it should be examined for use in $\mathrm{HF}$ patients. Such a diet is typically low in SFA, with increased consumption of low-fat dairy products, complex carbohydrate, fish and vegetables ${ }^{(51)}$. This dietary pattern is in contrast to that of the UK population, which typically consumes a diet higher in refined carbohydrate and SFA and lower in vegetables ${ }^{(54)}$. If individuals with HF are required to change their diet, support and guidance to the most appropriate way of achieving an optimal nutrient intake should be provided.

Hummel et al. ${ }^{(55)}$ demonstrated a significant improvement in ventricular diastolic function in thirteen patients with $\mathrm{HFpEF}$ when these patients were provided with a Na-restricted DASH diet $(50 \mathrm{mmol} / 8786 \mathrm{~kJ}(2100 \mathrm{kcal}))$. Specifically, adherence to this dietary pattern improved EF by $8 \%$ and increased stroke volume by approximately $11 \%$. Although impressive, the relatively small sample size and feeding protocol (controlled feeding with prepared meals) mean that such a finding may not be observed in free-living individuals with HF. In addition, the nature of the population studied means that this finding may also be only linked to those with HTN and HFpEF (Table 3). The Geriatric Out of Hospital Randomised Meal Trial in Heart Failure is one such study that will address whether such findings can be reproduced using a home-delivered low-Na meal, examining quality of life and cardiac functional parameters, although this study itself is still limited by the provision of meals ${ }^{(56)}$

Levitan et al. ${ }^{(57)}$ studied women enrolled in the Women's Health Initiative who were admitted to hospital with HF to identify whether adherence to a Mediterranean or DASH diet pattern influenced CVD mortality. After a median of 4.6 years of follow-up, there were 1385/3215 deaths following HF hospitalisation. When stratified into quartiles, greater adherence to either the Mediterranean or DASH diet was associated with a substantial reduction in the hazard rate (HR) associated with mortality. Specifically, the HR for death was 16 and 15\% lower in the DASH and Mediterranean diet group, respectively, although only reaching significance in the DASH group. Further analysis of the dietary intake of either Mediterranean or DASH patients revealed that greater adherence to each diet was associated with increased consumption of fruit and vegetables, nuts, legumes, whole grains and fish, and reduced intake of sweetened beverages and red and processed meat. However, important limitations of this study were acknowledged by the authors, including difficulty in recording $\mathrm{Na}$, fluid and olive oil intakes, in addition to the group comprising those diagnosed with HFpEF. Although the results may be promising for the DASH diet, they do not support the advocacy for the Mediterranean-style diet, despite a favourable trend. However, previous crosssectional data have shown that adherence to a Mediterranean Diet is associated with improved diastolic function in individuals with congestive $\mathrm{HF}^{(58)}$ (Table 3), and subsequent studies have shown the Mediterranean diet to reduce HF biomarkers in individuals at high-risk CVD ${ }^{(59)}$. Therefore, at present, the role of the Mediterranean diet in the management of HF remains to be fully examined. There is a clear need for large, randomised trials investigating whether the improvement in mortality rate observed in the DASH group is driven by the restriction in $\mathrm{Na}$ or a rather combined effect of diet and $\mathrm{Na}$ restriction, and whether the Mediterranean diet has a role in the management of HF.

\section{Low carbohydrate and high protein}

There are several interesting reports regarding the use of lowcarbohydrate diets in humans with HF. However, an important limitation of some of these studies cited is that they are almost exclusively conference abstracts, and thus caution should be exercised when interpreting them. Nonetheless, in patients with $\mathrm{HF}$ and right-ventricular dysfunction, a diet classified as low in carbohydrate ( $40 \%$ carbohydrate, $40 \%$ fat, $20 \%$ protein) has been shown to be effective at increasing weight loss and improving $\mathrm{O}_{2}$ saturation when compared with a conventional diet containing $50 \%$ of energy as carbohydrate ${ }^{(60)}$. In addition, the authors report an improvement in HF functional class. Similar to many HF trials, the study suffered from a relatively small sample size and short duration, including twenty-one individuals studied for a duration of 2 months. Therefore, the long-term consequences of such a pattern remain unknown in HF patients. Importantly, this study highlights a key issue facing nutritional interventions: how diets are defined; $40 \%$ energy as carbohydrate may be regarded by many as not being 'low carbohydrate' and is consistent with that achieved in the Prevención con Dieta Mediterránea (PREDIMED) study ${ }^{(61)}$ (widely defined as a Mediterranean diet). It would be appropriate for the The National Heart, Lung, and Blood Institute and National Institutes of Health Office of Dietary Supplements Working Group $^{(62)}$ to also consider a standard protocol for reporting the nutritional composition of experimental diets in HF studies to facilitate greater comparison of dietary interventions, in addition to their other current recommendations (Table 3).

Modifying protein intake has been shown to be effective in reducing weight in obese patients (mean BMI $37 \cdot 3 \mathrm{~kg} / \mathrm{m}^{2}$ ) with NYHA class II-III HF. Evangelista et al. ${ }^{(63)}$ compared a 12-week hypoenergetic diet $(5021-6276 \mathrm{~kJ} / \mathrm{d}(1200-1500 \mathrm{kcal} / \mathrm{d}))$ containing (as percentage of energy) 30\% protein, $40 \%$ carbohydrate and $30 \%$ fat with a standard protein, hypoenergetic diet (55\% total energy from carbohydrates, $15 \%$ from protein and $30 \%$ from fat) or the recommendations by the AHA. The authors noted that the high protein hypoenergetic diet led to a 


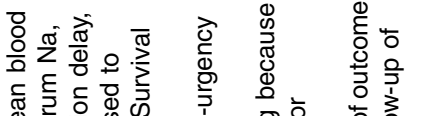

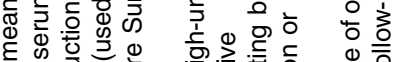

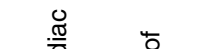

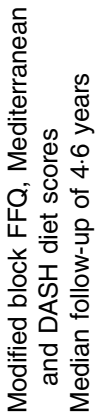

盇

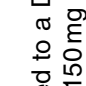

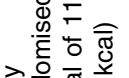

글흄 땅음

का

突点素

这.

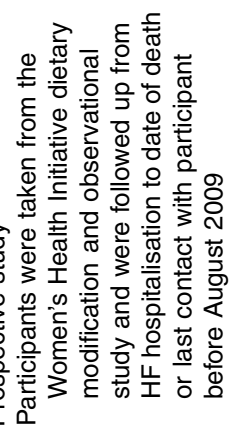

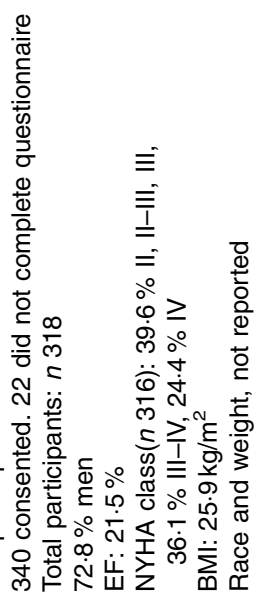

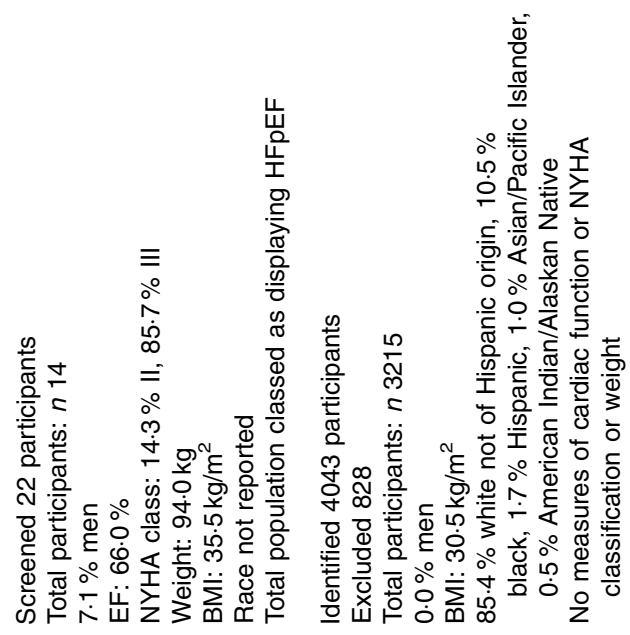

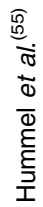




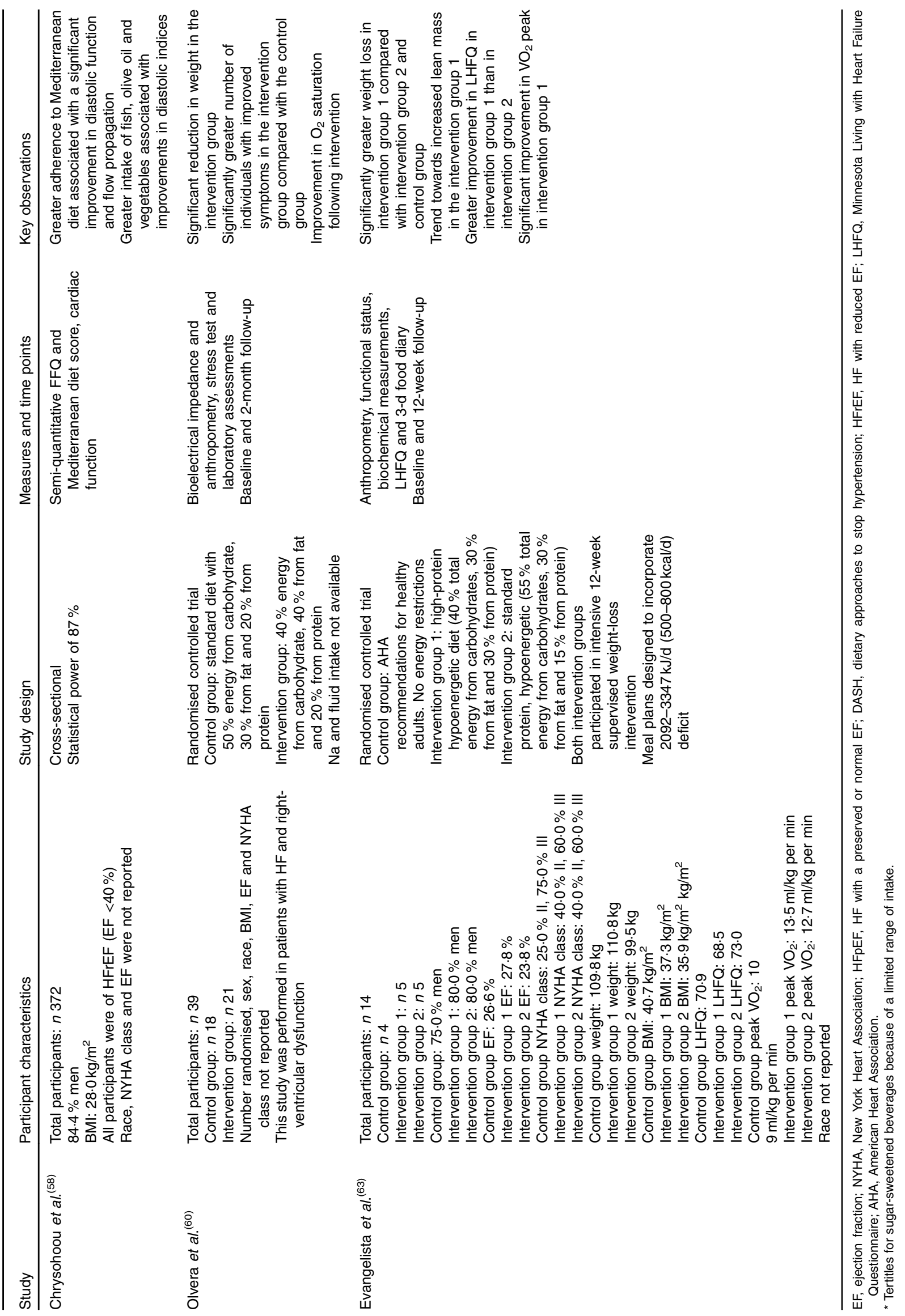



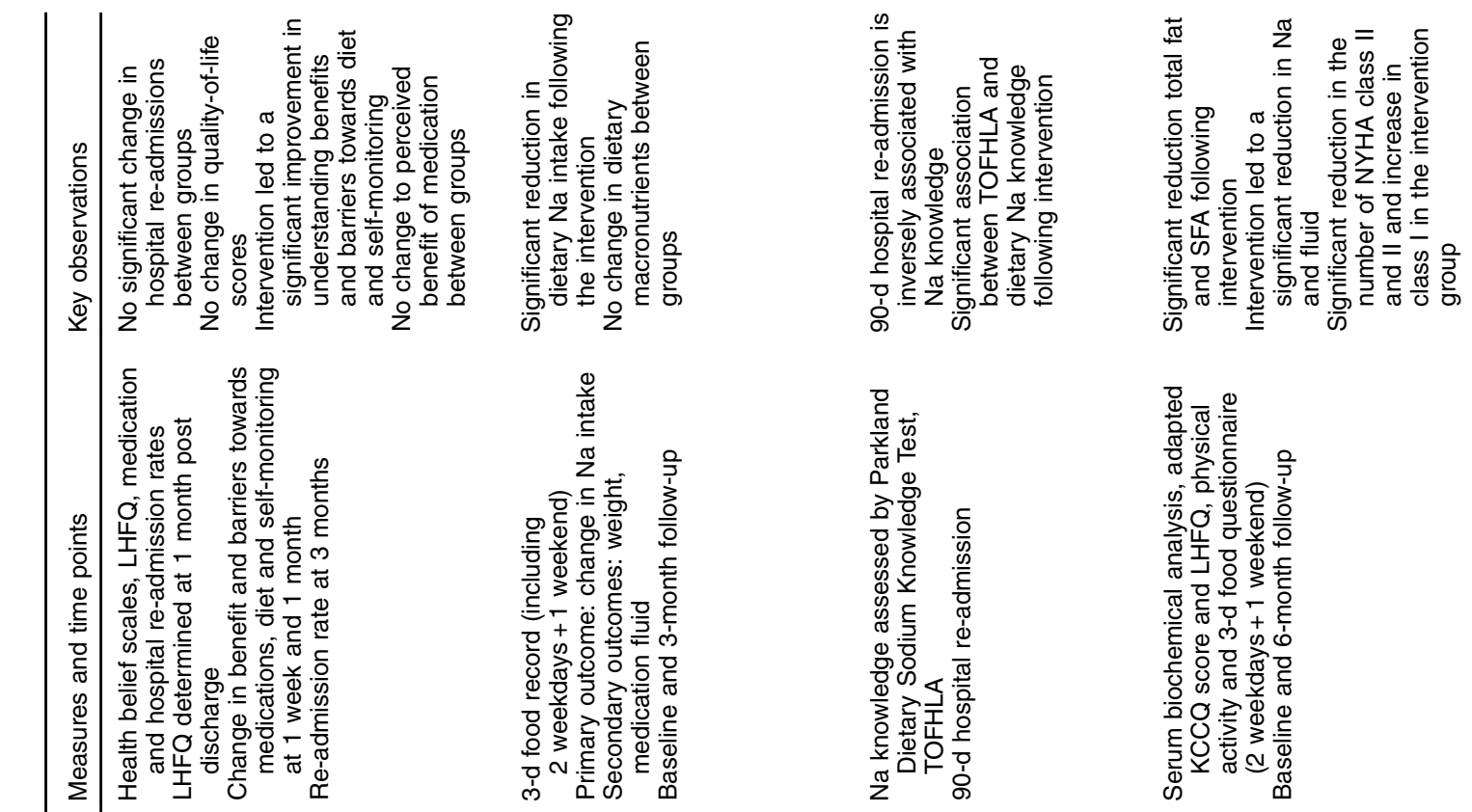

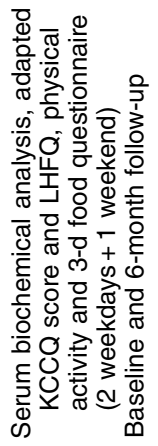
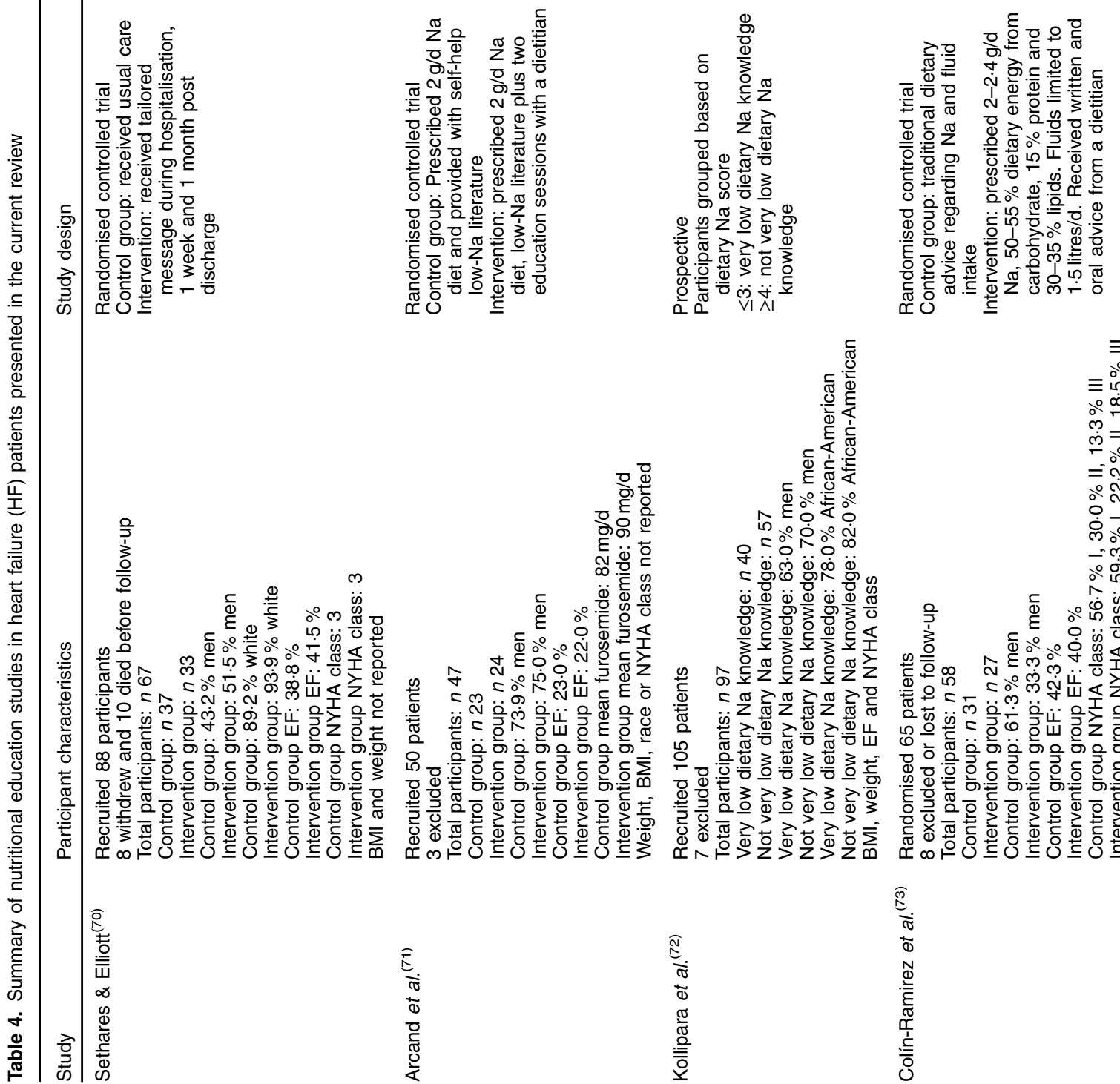

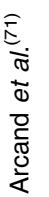
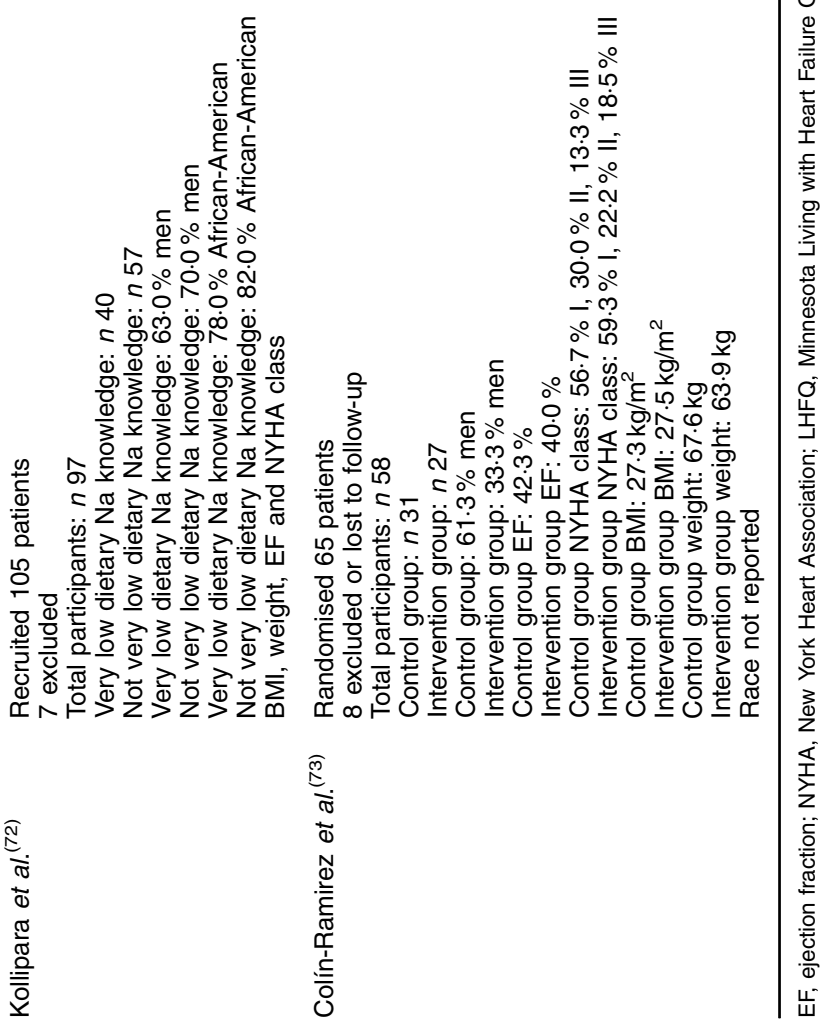
greater reduction in percentage of body fat and improved the patient's quality of life (assessed by the Minnesota Living with Heart Failure Questionnaire). However, this study was performed in five individuals, and it is therefore severely limited by the small sample size (Table 3). At present, there are no available large-scale dietary trails investigating protein intake and cardiac structure and function, functional status and quality of life in HF patients, although these are in development ${ }^{(64)}$.

\section{The obesity paradox}

Studies by Chrysohoou et al. ${ }^{(58)}$ and Estruch et al. ${ }^{(61)}$ suggest a beneficial effect of weight loss in HF patients; however, it is important to recognise that uncontrolled weight loss in HF is linked with increased incidence of mortality ${ }^{(3)}$. The importance of weight in HF patients has frequently been examined as part of the obesity paradox. The obesity paradox refers to observations that link the presence of obesity (and in some instances overweight) in HF patients with improved survival in comparison with lean counterparts. Horwich et $a l .^{(65)}$ was one of the first groups to demonstrate the inverse relationship between weight and mortality in patients with HF. In this study, the majority of participants were of NYHA class IV and had an EF of $22 \%$, with obese patients more likely to have diabetes and HTN. Following multivariate analysis, overweight and obesity were found to be associated with a significant survival benefit at 2 years, with the worst prognosis seen in those who were underweight, followed by those who were classified as recommended weight. Importantly, although this study is used to draw evidence to the protective nature of obesity, the survival benefit was not evident at the 5 -year follow-up. In addition, categorisation of patients as underweight at baseline may not have accounted for unintentional weight loss before the study. Importantly, this study was only performed in individuals with HFrEF, and therefore it may not apply to those with HFpEF. Despite this, subsequently larger meta-analysis studies have further reinforced this observation. Oreopoulos et al. ${ }^{(66)}$ analysed a total of nine observational studies demonstrating that both overweight and obesity were associated with a reduced relative risk of all-cause and cardiovascular mortality when compared with patients with normal BMI levels. Regrettably, the authors of this study did not extract data on EF; however, a more recent a meta-analysis examined whether HF subtype (HFrEF $v$. HFpEF) affected the obesity paradox. Using individual patient data, Padwal et al. ${ }^{(67)}$ demonstrated the existence of a U-shaped relationship between BMI and all-cause death in both HFrEF and HFpEF patients. In patients with HFrEF or HFpEF, the lowest hazard ratio for all-cause mortality was observed when comparing those individuals with a BMI between 30 and $34.9 \mathrm{~kg} / \mathrm{m}^{2}$ against the reference BMI range of $22.5-24.9 \mathrm{~kg} / \mathrm{m}^{2}$. In both subtypes, a $\mathrm{BMI}<22.5 \mathrm{~kg} / \mathrm{m}^{2}$ was associated with a higher risk of all-cause death.

There may be several mechanisms behind the proposed obesity paradox in HF. It is well known that advanced HF is associated with cachexia ${ }^{(8)}$, and in this regard greater adiposity may simply reflect greater body energy stores and hence greater resistance to the metabolic changes associated with the cachexic state. As shown by Padwal et al. ${ }^{(67)}$, individuals who were obese were also more likely to be receiving cardiovascular medication, potentially suggesting greater clinical input and therefore greater clinical management of their condition. However, it should be noted that this was adjusted for in their study with no effect upon their findings. Also, the use of BMI as a marker of fatness in $\mathrm{HF}$ has been questioned, with more accurate measurements of body composition being proposed $^{(68)}$. The presence of the obesity paradox means that we may need to re-examine advice to achieve a healthy weight in HF patients, and it raises important questions regarding the role of weight loss ${ }^{(60,63)}$ on the outcome of mortality. There may be a point at which excess weight is not associated with any additional benefit but conversely increases risk. Indeed, in morbidly obese $\left(\mathrm{BMI} \geq 40 \mathrm{~kg} / \mathrm{m}^{2}\right) \mathrm{HF}$ patients, the obesity paradox is absent ${ }^{(69)}$. Therefore, one may conclude that in those individuals with morbid obesity intentional weight loss may be beneficial in terms of reducing mortality rate; however, this should be carefully monitored and controlled. In lower-BMI categories, a reduction in weight may improve clinical symptoms and disease classification, but it may have a negative impact on long-term survival. It would be useful for future studies examining the relationship between body weight and HF mortality to assess adipose tissues deposits (both visceral and subcutaneous) and lean mass, in addition to cardiorespiratory fitness following weight loss.

\section{Nutritional messages: the role of the dietitian}

A key aspect of implementing a dietary strategy is addressing pre-conceived ideas and beliefs regarding nutrition. A tailored nutritional message to patients with $\mathrm{HF}$ is sufficient to alter patients' views and attitudes towards medications, adherence to a Na-restricted diet and self-monitoring ${ }^{(70)}$. Further support for the importance of nutritional input can be derived from Arcand et $a l .{ }^{(71)}$. In this 3-month study, HF patients randomised to a dietitian-led education group showed greater improvements in salt reduction in comparison with usual care (self-help literature). Although such a frequent dietetic input may be unlikely in the current health-care setting, clinicians reviewing their patients may wish to follow-up nutritional advice and reinforce nutritional messages at every opportunity. Indeed, frequent nutritional counselling with HF patients may improve knowledge surrounding foods and reduce admissions. In HF patients, a low level of Na knowledge has been shown to be associated with a significantly greater OR for hospital re-admission for $\mathrm{HF}^{(72)}$. Using the Test of Functional Health Literacy in Adults tool, Na knowledge was associated with a low health literacy score. When nutritional interventions are combined with appropriate educational session, substantial improvement in quality of life and disease score can be seen. For example, a nutritional intervention consisting of $2000-2400 \mathrm{mg} / \mathrm{d} \mathrm{Na}, 50-55 \%$ (as percentage of energy) carbohydrate, $15 \%$ protein, $<10 \%$ SFA, $15 \%$ MUFA and $10 \%$ PUFA coupled with written and oral instructions from a dietitian led to a significant improvement in HF classification and quality of life when compared with a control group receiving general 
nutritional advice ${ }^{(73)}$. Indeed, the improvement in HF classification was reflected by a significant reduction in the number of individuals with NYHA class II and III and an increase in the number of those with class I by the end of the study (Table 4).

As such, this would suggest that by using appropriate methods of patient education and trained individuals, it is never too late to make important and significant dietary changes that may improve quality of life.

\section{Discussion and conclusions}

HF remains a chronic and debilitating condition. Although the value of dietary manipulation is well known in the primary, secondary and tertiary prevention of CVD, it is undervalued in patients with HF and is reflected by the paucity of data in guidelines. Despite a large body of experimental data produced from animal models of HF examining the effect of different diet compositions, this has not translated into human trials. From animal trials, it is clear that the traditional demonisation of fat may not be justified in HF, and human studies should be designed to evaluate the therapeutic effectiveness of cardioprotective fats in HF. Within this, consideration should be given to the underlying HF aetiology in addition to other comorbidities. Indeed, by manipulating dietary nutrient composition, it is possible for those individuals with other comorbidities to benefit from the potential therapeutic nature of food.

Studies that have been published in this field - albeit largely observational - now suggest that diet advice in this area may need to be re-examined, with the traditional cardioprotective diets such as the Mediterranean and DASH potentially being of benefit. Such diet patterns have been shown to increase the consumption of cardioprotective food items such as fruit and vegetables, nuts, legumes, whole grains and fish and are likely to have additional health effects beyond $\mathrm{HF}$.

It is simple to decide what foods an individual should consume, yet much more difficult to actually achieve this. Regular nutritional education has been shown to lead to better adoption of a prescribed diet and may lead to improved overall nutritional status. In some studies, this has also translated to improvements in quality of life and reduced severity of symptoms when delivered by nutritionally trained individuals. The feasibility of such a means of improving nutritional knowledge is clearly in need of evaluation, given the potential cost such a service may incur.

Although the studies presented in this review are promising, many are limited by small sample sizes, short duration and observational study design. It is therefore a requirement that in order to progress towards better evidence-based dietary advice for patients with HF, larger, longer, randomised clinical trials are needed. Such studies should account for differences in HF subtype (HFrEF $v$. HFpEF) and have clearly defined clinical end points. In addition, there is a requirement for standardisation of dietary reporting. The studies highlighted in this review provide a potential starting point for the development of future trials, and fundamentally demonstrate that, in addition to fluid and $\mathrm{Na}$, consideration should be given to other dietary components.

\section{Acknowledgements}

The author thanks their colleagues for interesting and stimulating discussions.

The present review received no financial support

All literature was searched for, analysed and revisions made by the author.

The author declares no conflicts of interest that may undermine the validity of the conclusions made by this work.

\section{References}

1. McMurray JJ, Adamopoulos S, Anker SD, et al. (2012) ESC guidelines for the diagnosis and treatment of acute and chronic heart failure 2012: the task force for the diagnosis and treatment of acute and chronic heart failure 2012 of the European Society of Cardiology. Developed in collaboration with the Heart Failure Association (HFA) of the ESC. Eur Heart $J$ 33, 787-847.

2. Cleland J, Dargie H, Hardman S, et al. (2013) National heart failure audit April 2012-March 2013. http://www.ucl.ac. uk/nicor/audits/heartfailure/documents/annualreports/hfann ual12-13.pdf (accessed May 2015).

3. Mitchell P, Marle D, Donkor A, et al. (2015) National heart failure audit April 2013-March 2014. http://www.ucl.ac. uk/nicor/audits/heartfailure/documents/annualreports/hfann ual13-14.pdf (accessed November 2015).

4. Lam CSP, Donal E, Kraigher-Krainer E, et al. (2011) Epidemiology and clinical course of heart failure with preserved ejection fraction. Eur J Heart Fail 13, 18-28.

5. Borlaug BA. (2013) Heart failure with preserved and reduced ejection fraction: different risk profiles for different diseases. Eur Heart J 34, 1393-1395.

6. Ather S, Chan W, Bozkurt B, et al. (2012) Impact of noncardiac comorbidities on morbidity and mortality in a predominantly male population with heart failure and preserved versus reduced ejection fraction. J Am Coll Cardiol 59, 998-1005.

7. Rossignol P, Masson S, Barlera S, et al. (2015) Loss in body weight is an independent prognostic factor for mortality in chronic heart failure: insights from the GISSI-HF and Val-HeFT trials. Eur J Heart Fail 17, 424-433.

8. Rahman A, Jafry S, Jeejeebhoy K, et al. (2015) Malnutrition and cachexia in heart failure. JPEN J Parenter Enteral Nutr (Epublication ahead of print version 29 January 2015).

9. Witte KKA, Clark AL \& Cleland JGF (2001) Chronic heart failure and micronutrients. J Am Coll Cardiol 37, 1765-1774

10. Yancy CW, Jessup M, Bozkurt B, et al. (2013) 2013 ACCF/AHA guideline for the management of heart failure: a report of the American College of Cardiology Foundation/American Heart Association task force on practice guidelines. Circulation $\mathbf{6 2}$, 1495-1539.

11. Grossman W (1980) Cardiac hypertrophy: useful adaptation or pathologic process? Am J Med 69, 576-584.

12. Oie E, Bjornerheim R, Clausen OP, et al. (2000) Cyclosporin A inhibits cardiac hypertrophy and enhances cardiac dysfunction during postinfarction failure in rats. Am J Physiol Heart Circ Physiol 278, H2115-H2123.

13. Shiojima I, Sato K, Izumiya Y, et al. (2005) Disruption of coordinated cardiac hypertrophy and angiogenesis contributes to the transition to heart failure. J Clin Invest 115, 2108-2118.

14. Velagaleti RS, Gona P, Pencina MJ, et al. (2014) Left ventricular hypertrophy patterns and incidence of heart failure with 
preserved versus reduced ejection fraction. J Am Coll Cardiol 113, $117-122$.

15. Stanley WC, Recchia FA \& Lopaschuk GD (2005) Myocardial substrate metabolism in the normal and failing heart. Physiol Rev 85, 1093-1129.

16. Kolwicz SC Jr, Purohit S \& Tian R (2013) Cardiac metabolism and its interactions with contraction, growth, and survival of cardiomyocytes. Circ Res 113, 603-616.

17. Doenst T, Nguyen TD \& Abel ED (2013) Cardiac metabolism in heart failure: implications beyond ATP production. Circ Res 113, 709-724.

18. Kato T, Niizuma S, Inuzuka Y, et al. (2010) Analysis of metabolic remodeling in compensated left ventricular hypertrophy and heart failure. Circ Heart Fail 3, 420-430.

19. de Brouwer KF, Degens H, Aartsen WM, et al. (2006) Specific and sustained down-regulation of genes involved in fatty acid metabolism is not a hallmark of progression to cardiac failure in mice. J Mol Cell Cardiol 40, 838-845.

20. Abdurrachim D, Luiken JJ, Nicolay K, et al. (2015) Good and bad consequences of altered fatty acid metabolism in heart failure: evidence from mouse models. Cardiovasc Res 106, 194-205.

21. Sack MN, Rader TA, Park S, et al. (1996) Fatty acid oxidation enzyme gene expression is downregulated in the failing heart. Circulation 94, 2837-2842.

22. Neubauer S (2007) The failing heart - an engine out of fuel. $N$ Engl J Med 356, 1140-1151.

23. Patten RD \& Hall-Porter MR (2009) Small animal models of heart failure. Circ Heart Fail 2, 138-144.

24. Berry JM, Naseem RH, Rothermel BA, et al. (2007) Models of cardiac hypertrophy and transition to heart failure. Drug Discov Today Dis Models 4, 197-206.

25. Stanley WC, Dabkowski ER, Ribeiro RF, et al. (2012) Dietary fat and heart failure: moving from lipotoxicity to lipoprotection. Circ Res 110, 764-776.

26. Berthiaume JM, Bray MS, McElfresh TA, et al. (2010) The myocardial contractile response to physiological stress improves with high saturated fat feeding in heart failure. Am J Physiol Heart Circ Physiol 299, H410-H421.

27. Berthiaume JM, Young ME, Chen X, et al. (2012) Normalizing the metabolic phenotype after myocardial infarction: impact of subchronic high fat feeding. J Mol Cell Cardiol 53, 125-133.

28. Tuunanen H, Engblom E, Naum A, et al. (2006) Free fatty acid depletion acutely decreases cardiac work and efficiency in cardiomyopathic heart failure. Circulation 114, 2130-2137.

29. Banke NH, Wende AR, Leone TC, et al. (2010) Preferential oxidation of triacylglyceride-derived fatty acids in heart is augmented by the nuclear receptor PPARalpha. Circ Res 107, 233-241.

30. Wende AR, Symons JD \& Abel ED (2012) Mechanisms of lipotoxicity in the cardiovascular system. Curr Hypertens Rep 14, 517-531.

31. van Bilsen $M$ \& Planavila A (2014) Fatty acids and cardiac disease: fuel carrying a message. Acta Physiol 211, 476-490.

32. Listenberger LL, Han X, Lewis SE, et al. (2003) Triglyceride accumulation protects against fatty acid-induced lipotoxicity. Proc Natl Acad Sci U S A 100, 3077-3082.

33. Greenberg AS, Coleman RA, Kraemer FB, et al. (2011) The role of lipid droplets in metabolic disease in rodents and humans. J Clin Invest 121, 2102-2110.

34. O'Donnell JM, Fields AD, Sorokina N, et al. (2008) The absence of endogenous lipid oxidation in early stage heart failure exposes limits in lipid storage and turnover. $J$ Mol Cell Cardiol 44, 315-322.

35. Lahey R, Wang X, Carley AN, et al. (2014) Dietary fat supply to failing hearts determines dynamic lipid signaling for nuclear receptor activation and oxidation of stored triglyceride. Circulation 130, 1790-1799.

36. Tavazzi L, Maggioni AP, Marchioli R, et al. (2008) Effect of $n-3$ polyunsaturated fatty acids in patients with chronic heart failure (the GISSI-HF trial): a randomised, double-blind, placebo-controlled trial. Lancet 372, 1223-1230.

37. Xin W, Wei W \& Li X (2012) Effects of fish oil supplementation on cardiac function in chronic heart failure: a meta-analysis of randomised controlled trials. Heart 98, 1620-1625.

38. Rizos EC, Ntzani EE, Bika E, et al. (2012) Association between omega-3 fatty acid supplementation and risk of major cardiovascular disease events: a systematic review and metaanalysis. JAMA 308, 1024-1033.

39. Chowdhury R, Warnakula S, Kunutsor S, et al. (2014) Association of dietary, circulating, and supplement fatty acids with coronary risk: a systematic review and meta-analysis. Ann Intern Med 160, 398-406.

40. Djoussé L, Akinkuolie AO, Wu JH, et al. (2012) Fish consumption, omega-3 fatty acids and risk of heart failure: a meta-analysis. Clin Nutr 31, 846-853.

41. Bansal S, Lindenfeld J \& Schrier RW (2009) Sodium retention in heart failure and cirrhosis potential role of natriuretic doses of mineralocorticoid antagonist? Circ Heart Fail 2, 370-376.

42. Weinberger MH, Fineberg NS, Fineberg SE, et al. (2001) Salt sensitivity, pulse pressure, and death in normal and hypertensive humans. Hypertension 37, 429-432.

43. Travers B, O'Loughlin C, Murphy NF, et al. (2007) Fluid restriction in the management of decompensated heart failure: no impact on time to clinical stability. J Card Fail 13, 128-132.

44. Aliti GB, Rabelo ER, Clausell N, et al. (2013) Aggressive fluid and sodium restriction in acute decompensated heart failure: a randomized clinical trial. JAMA Intern Med 173, 1058-1064.

45. Colín-Ramirez E, McAlister FA, Zheng Y, et al. (2015) The long-term effects of dietary sodium restriction on clinical outcomes in patients with heart failure. The SODIUM-HF (Study of Dietary Intervention Under $100 \mathrm{mmol}$ in Heart Failure): a pilot study. Am Heart J 169, 274-281.

46. Paterna S, Fasullo S, Parrinello G, et al. (2011) Short-term effects of hypertonic saline solution in acute heart failure and long-term effects of a moderate sodium restriction in patients with compensated heart failure with New York Heart Association class III (Class C) (SMAC-HF Study). Am J Med Sci 342, 27-37.

47. Wessler JD, Hummel SL \& Maurer MS (2014) Dietary interventions for heart failure in older adults: re-emergence of the hedonic shift. Prog Cardiovasc Dis 57, 160-167.

48. Jefferson K, Ahmed M, Choleva M, et al. (2015) Effect of a sodium-restricted diet on intake of other nutrients in heart failure: implications for research and clinical practice. J Card Fail 21, 959-962.

49. Graudal NA, Hubeck-Graudal T \& Jürgens G (2012) Effects of low-sodium diet vs. high-sodium diet on blood pressure, renin, aldosterone, catecholamines, cholesterol, and triglyceride (Cochrane Review). Am J Hypertens 25, 1-15.

50. Masson S, Solomon S, Angelici L, et al. (2010) Elevated plasma renin activity predicts adverse outcome in chronic heart failure, independently of pharmacologic therapy: data from the Valsartan Heart Failure Trial (Val-HeFT). J Card Fail 16, 964-970.

51. Appel LJ, Moore TJ, Obarzanek E, et al. (1997) A clinical trial of the effects of dietary patterns on blood pressure. $N$ Engl $J$ Med 336, 1117-1124.

52. Spaderna H, Zahn D, Pretsch J, et al. (2013) Dietary habits are related to outcomes in patients with advanced heart failure awaiting heart transplantation. J Card Fail 19, 240-250. 
53. Levitan EB, Wolk A \& Mittleman MA (2009) Relation of consistency with the dietary approaches to stop hypertension diet and incidence of heart failure in men aged 45 to 79 years. Am J Cardiol 104, 1416-1420.

54. Bates B, Lennox A, Prentice A, et al.2014) National diet and nutrition survey. Results from years 1,2, 3 and 4 (combined) of the Rolling Programme (2008/2009-2011/2012). Crown Copyright. https://www.gov.uk/government/uploads/system/ uploads/attachment_data/file/310995/NDNS_Y1_to_4_UK_re port.pdf (accessed May 2015).

55. Hummel SL, Seymour EM, Brook RD, et al. (2013) LOw-sodium DASH diet improves diastolic function and ventricular-arterial coupling in hypertensive heart failure with preserved ejection fraction. Circ Heart Fail 6, 1165-1171.

56. University of Michigan (2014) Effects of home-delivered lowsodium meals in older adults following heart failure hospitalization. https://clinicaltrials.gov/ct2/show/NCT02148679: NML Identifier NCT02148679 (accessed June 2015).

57. Levitan EB, Lewis CE, Tinker LF, et al. (2013) Mediterranean and DASH diet scores and mortality in women with heart failure: the Women's Health Initiative. Circ Heart Fail 6, $1116-1123$

58. Chrysohoou C, Pitsavos C, Metallinos G, et al. (2012) Crosssectional relationship of a Mediterranean type diet to diastolic heart function in chronic heart failure patients. Heart Vessels 27, 576-584.

59. Fitó M, Estruch R, Salas-Salvadó J, et al. (2014) Effect of the Mediterranean diet on heart failure biomarkers: a randomized sample from the PREDIMED trial. Eur J Heart Fail 16, 543-550.

60. Olvera G, Castillo L, Orea A, et al. (2014) PP125-SUN: effect of a low carbohydrate diet on the clinical status of patients with heart failure and right ventricular dysfunction. Clin Nutr 33, S66.

61. Estruch R, Ros E, Salas-Salvadó J, et al. (2013) Primary prevention of cardiovascular disease with a Mediterranean diet. N Engl J Med 368, 1279-1290.

62. NIH Heart, Lung and Blood Institute (NHLBI) \& NHLBI Working Group (2013) Designing clinical studies to evaluate the role of nutrition and diet in heart failure management. http://www.nhlbi.nih.gov/research/reports/2013-heart-failuremanagement (accessed June 2015).
63. Evangelista LS, Heber D, Li Z, et al. (2009) Reduced body weight and adiposity with a high-protein diet improves functional status, lipid profiles, glycemic control, and quality of life in patients with heart failure: a feasibility study. Eur $J$ Cardiovasc Nurs 24, 207-215.

64. Motie M, Evangelista LS, Horwich T, et al. (2013) Pro-HEART-a randomized clinical trial to test the effectiveness of a high protein diet targeting obese individuals with heart failure: rationale, design and baseline characteristics. Contemp Clin Trials 36, 371-381.

65. Horwich TB, Fonarow GC, Hamilton MA, et al. (2001) The relationship between obesity and mortality in patients with heart failure. J Am Coll Cardiol 38, 789-795.

66. Oreopoulos A, Padwal R, Kalantar-Zadeh K, et al. (2008) Body mass index and mortality in heart failure: a meta-analysis. $\mathrm{Am}$ Heart J 156, 13-22.

67. Padwal R, McAlister FA, McMurray JJV, et al. (2014) The obesity paradox in heart failure patients with preserved versus reduced ejection fraction: a meta-analysis of individual patient data. Int J Obesity 38, 1110-1114.

68. Oreopoulos A, Fonarow GC, Ezekowitz JA, et al. (2011) Do anthropometric indices accurately reflect directly measured body composition in men and women with chronic heart failure? Congest Heart Fail 17, 89-91.

69. Nagarajan V, Cauthen CA, Starling RC, et al. (2013) Prognosis of morbid obesity patients with advanced heart failure. Congest Heart Fail 19, 160-164.

70. Sethares KA \& Elliott K (2004) The effect of a tailored message intervention on heart failure readmission rates, quality of life, and benefit and barrier beliefs in persons with heart failure. Heart Lung 33, 249-260.

71. Arcand JA, Brazel S, Joliffe C, et al. (2005) Education by a dietitian in patients with heart failure results in improved adherence with a sodium-restricted diet: a randomized trial. Am Heart J 150, 716.e1-716.e5.

72. Kollipara UK, Jaffer O, Amin A, et al. (2008) Relation of lack of knowledge about dietary sodium to hospital readmission in patients with heart failure. Am J Cardiol 102, 1212-1215.

73. Colín-Ramirez E, Castillo ML, Orea TA, et al. (2004) Effects of a nutritional intervention on body composition, clinical status, and quality of life in patients with heart failure. Nutrition $\mathbf{2 0}$, 890-895. 